\title{
Outcome of Pregnancy in the Era of PEGylated Interferon- $\alpha 2 a$ in Females with Chronic Myeloid Leukemia: An Experience from Qatar
}

\author{
Mohammad Abu-Tineha Nancy Kassem ${ }^{b}$ \\ Mohammad Abdul-Jaber Abdulla ${ }^{c}$ Omar Mohammad Ismail ${ }^{c}$ \\ Khaldun Obeidat $^{a}$ Rola Ghasoub ${ }^{b}$ Mohamed A. Yassin ${ }^{c}$ \\ aDepartment of Medical Education, Hamad Medical Corporation, Doha, Qatar; \\ bNational Center for Cancer Care and Research, Department of Pharmacy, Doha, Qatar; \\ 'National Center for Cancer Care and Research, Department of Hematology and BMT, \\ Doha, Qatar
}

\author{
Keywords \\ Chronic myeloid leukemia · Interferon · PEGylated interferon · PEG-INF- $22 a \cdot$ Pregnancy
}

\section{Abstract}

Chronic myelogenous leukemia (CML), also known as chronic myeloid leukemia, is a myeloproliferative neoplasm characterized by increased proliferation of the granulocytic cell line without loss of its capacity to differentiate. It accounts for $20 \%$ of all adults affected by leukemia. Tyrosine kinase inhibitors revolutionized the treatment for CML and improved quality of life. Fertility is an important issue for both males and females. Here, we report our experience with a pregnant female with $\mathrm{CML}$, and shed light on safety and efficacy of PEGylated interferon- $\alpha$ a in pregnant women with $\mathrm{CML}$ and its outcome.

\section{Introduction}

Chronic myelogenous leukemia (CML) is a myeloproliferative neoplasm characterized by the $B C R-A B L$ oncogene, which results from a reciprocal $t(9 ; 22)$ chromosomal translocation. CML constitutes $15 \%$ of adult leukemia. Incidence rates vary from 0.6 to 2.0 cases per 100,000 persons, increase with age and are higher in men than in women. The average age at diagnosis of CML is around 64 years [1]. However, CML can affect any age group. In fact, approximately $17 \%$ of cases occur in the age group of 20-44 years. Fertility in CML patients receiving tyrosine 
Abu-Tineh et al.: Outcome of Pregnancy with PEG-IFN- $\alpha 2 a$ in CML Females

kinase inhibitors (TKIs) has been addressed in certain studies, which concluded that, in male patients with CML receiving TKIs, there is a notable decrease in sperm parameters and decreased concentrations of serum T, LH, and FSH [2].

On the other hand, despite the advancement in treatment options, we still have limited data on the safety of TKIs in pregnancy and their effect on fertility. There remains a concern for the occurrence of rare congenital malformations and spontaneous abortions in association with TKI therapy, mainly with imatinib $[3,16]$. Management in pregnant females with CML remains challenging for both, patient and physician, given the risks on the fetus upon continuing the therapy versus the patient risk of withholding the treatment and potentially losing optimal disease response [3].

\section{Case Presentation}

A 43-year-old Filipino female patient, diagnosed with CML (chronic phase) was started on dasatinib as upfront therapy, and achieved complete hematologic, cytogenetic and molecular major response as per the ELN (European leukemia net) recommendations (2013). The patient got pregnant while on dasatinib, which mandated its immediate stoppage.

Alternatives were discussed with the patient: (1) to start with conventional interferon (safe and recommended); (2) to start with PEGylated interferon, but there is no data confirming its safety in pregnancy; (3) to take neither interferon nor TKIs, but this is a risky approach since the patient can progress to either an accelerated phase or blast crisis as a worst case scenario or remain in the chronic phase, which would be the best scenario, but this is not guaranteed. The patient and her husband opted for PEGylated interferon. She was referred to a high-risk pregnancy unit in the maternity hospital for close followup. Follow-up throughout pregnancy showed a normal fetus with no evidence of teratogenicity.

\section{Discussion}

The current management of pregnant patients with CML is a therapeutic challenge. Patients may initially present with CML while pregnant or may become pregnant while on active treatment.

Patients presenting with CML in the chronic phase must be assessed and are less likely considered for elective termination, even at the beginning of their pregnancy [4].

While in advanced phases (accelerated or blastic phases), the patient must be managed more aggressively and may need immediate intervention with TKIs. However, it is known that TKIs must not be used during pregnancy, especially during the first trimester, to consent the development of the organs.

Current treatment approaches include supportive care with interferon-alpha-2a (IFNa2a) and leukapheresis [5]. Leukapheresis is not a favored option due to its limited availability, complications and poor tolerance to its frequency [5].

IFN- $\alpha$ is considered safe in pregnancy [6]. It acts by controlling CML by directly inhibiting cell proliferation of the $\mathrm{Ph}+$ clone (protein synthesis, RNA breakdown), inducing an immune modulation, or eliciting a bone marrow microenvironment regulation of hematopoiesis [7].

It has been extensively studied as treatment for patients with CML resulting in hematologic remissions in the majority of patients treated with single-agent IFN-a [8-11].

On the other hand, interferon is known to cause significant side effects, such as fever, chills, and flu-like symptoms; in addition, it has a short half-life as it is barely detectable in the 
serum $24 \mathrm{~h}$ after its administration, requiring multiple frequent administration ( 2 or 3 times weekly) for sustained efficacy [12]. This makes it a less favorable option.

However, to overcome this limitation, 2 forms of PEGylated (covalent attachment of polyethylene glycol [Peg]) IFN- $\alpha$ have been developed: Peg-IFN- $\alpha 2 a$ and Peg-IFN- $\alpha 2 b$. The PEGylating resulted in different properties and pharmacokinetics, including sustained absorption/exposure and the prolonged half-life reduced clearance compared with IFN- $\alpha 2 \mathrm{a}$, allowing for once weekly doses $[12,13]$, attributing to better compliance with the medication.

Our patient had good compliance. She was followed up throughout her pregnancy at the high-risk pregnancy unit in the maternity hospital, and the outcome was a normal fetus with no teratogenicity.

\section{Conclusion}

PEG-INF might be the option for treatment of CML during pregnancy. So far, efficacy and safety of PEG-IFN in CML treatment have been investigated in several trials in combination with TKIs $[14,15]$, but it has not yet been investigated in pregnant women with CML.

\section{Acknowledgment}

We thank the Qatar National library for funding this article, and we thank the internal medicine residency program at Hamad Medical Corporation.

\section{Statement of Ethics}

Written informed consent was obtained from our patient to allow the publication of information.

\section{Disclosure Statement}

The authors have nothing to disclose.

\section{Funding Sources}

This article was funded by the Qatar national library.

\section{Authors Contributions}

Mohammad Abu-Tineh: writing the manuscript. Nancy Kassem, Mohammad Abdul-Jaber Abdulla, Omar Mohammad Ismail, Khaldun Obeidat, and Rola Ghasoub: clinical care. Mohamed A Yassin: writing and editing. 
Abu-Tineh et al.: Outcome of Pregnancy with PEG-IFN- $\alpha 2 a$ in CML Females

\section{References}

1 Yassin MA, Abdulla MA, Chandra P, Aldapt MB, Hamad Y, Akiki SJ, et al. Chronic Myeloid Leukemia in Adolescents and Young Adults: A Single Institute Experience. Blood. 2019;134:5915.

2 Yassin MA, Soliman AT, Sanctis VD. Effects of tyrosine kinase inhibitors on spermatogenesis and pituitary gonadal axis in males with chronic myeloid leukemia. J Cancer Res Ther. 2014;2:116-21.

3 Palani R, Milojkovic D, Apperley JF. Managing pregnancy in chronic myeloid leukemia. In: Chronic myeloid leukemia. Cham: Springer; 2016. p. 161-75.

4 Abruzzese E, Trawinska MM, de Fabritiis P, Baccarani M. Management of pregnant chronic myeloid leukemia patients. Expert Rev Hematol. 2016;9:(8):781-91.

5 Strobl FJ, Voelkerding KV, Smith EP. Management of chronic myeloid leukemia during pregnancy with leukapheresis. J Clin Apher The Official J Am Soc Apher. 1999;14(1):42-4.

6 Brojeni PY, Matok I, Bournissen FG, Koren G. A systematic review of the fetal safety of interferon alpha. Reprod Toxicol. 2012 Jun 1;33(3):265-8.

7 Dowding C, Gordon M, Guo AP, Maison D, Osterholz J, Siczkowski M, et al. Potential mechanisms of action of interferon- $\alpha$ in CML. Leuk Lymphoma. 1993 Jan 1;11(Suppl 1):185-91.

8 Talpaz M, McCredie KB, Mavligit GM, Gutterman JU. Leukocyte interferon-induced myeloid cytoreduction in chronic myelogenous leukemia. Blood. 1983;63(3):689-92.

9 Talpaz M, Kantarjian HM, McCredie K, Trujillo JM, Keating MJ, JU G. Hematologic remission and cytogenetic improvement induced by recombinant human interferon alpha A in chronic myelogenous leukemia. $\mathrm{N}$ Engl J Med. 1986 Apr 24;314(17):1065-9.

10 Bonifazi F, de Vivo A, Rosti G, Guilhot F, Guilhot J, Trabacchi E, et al. Chronic myeloid leukemia and interferon- $\alpha$ : a study of complete cytogenetic responders. Blood. 2001 Nov 15;98(10):3074-81.

11 Kantarjian HM, O’Brien S, Cortes JE, Shan J, Giles FJ, Rios MB, et al. Complete cytogenetic and molecular responses to interferon- $\alpha$-based therapy for chronic myelogenous leukemia are associated with excellent long-term prognosis. Cancer. 2003 Feb 15;97(4):1033-41.

12 Simonsson B, Gedde-Dahl T, Markevärn B, Remes K, Stentoft J, Almqvist A, et al. Combination of pegylated IFN- $\alpha 2 \mathrm{~b}$ with imatinib increases molecular response rates in patients with low-or intermediate-risk chronic myeloid leukemia. Blood. 2011 Sep 22;118(12):3228-35.

13 Yassin MA, Al-Dewik NI, ElAyoubi H, Cassinat B. Efficacy and safety of pegelated interferon alpha2a once monthlycompared toonceweeklydosein patients with essential thrombocythemia. Blood.2013;122(21):405454.

14 Preudhomme C, Guilhot J, Nicolini FE, Guerci-Bresler A, Rigal-Huguet F, Maloisel F, et al. Imatinib plus peginterferon alfa-2a in chronic myeloid leukemia. N Engl J Med. 2010 Dec 23;363(26):2511-21.

15 Guilhot F, Preudhomme C, Guilhot J, Mahon FX, Nicolini FE, Rigual-Huguet F, et al. Significant Higher Rates of Undetectable Molecular Residual Disease and Molecular Responses with Pegylated Form of Interferon a2a in Combination with Imatinib (IM) for the Treatment of Newly Diagnosed Chronic Phase (CP) Chronic Myeloid Leukaemia (CML) Patients (pts): Confirmatory Results at 18 Months of Part 1 of the Spirit Phase III Randomized Trial of the French CML Group (FI LMC). Blood. 2009;114(22):340.

16 Chelysheva E, Turkina A, Rea D, Rousselot P, Nicolini FE, Trawinska MM, et al. Pregnancy Outcome In Female Patients With Chronic Myeloid Leukemia Worldwide: Analysis Of 305 Cases Of The European Leukemia Net Registry: S881. HemaSphere. 2019 Jun;13:395-6. 